\title{
Diversidad biológica, distribución y estrategias de conservación de la ictiofauna de la cuenca del río Moctezuma, centro de México
}

\author{
Pedro Joaquín Gutiérrez-Yurrita*, José Alfredo Morales-Ortiz y Liliana Marín-García
}

Instituto Politécnico Nacional, Centro Interdisciplinario de Investigaciones y Estudios sobre Medio Ambiente y Desarrollo. Calle 30 de Junio de 1520 s/n, Col. La Laguna Ticomán. México D.F. 07340. México. Tel. (0052) 5557296000 exts. 52707 / 52708.

* Autor responsable de la correspondencia: pgutierrezy@ipn.mx

Recibido: $11 / 10 / 2012$

Aceptado: 22/03/2013

A la memoria del Maestro, Doctor Salvador Contreras Balderas (1936-2009)

\begin{abstract}
Biological diversity, distribution and conservation strategies of the fish in the Moctezuma River, Central México

In the Moctezuma river, Central Mexico we recorded thirty five species of fish, corresponding to a $\gamma$ diversity of 2.5 . The river basin is located in the biological transition zone between both America's bioregions. The highest $\alpha$ diversity was found in the ecotope Jalpilla $\left(H^{\prime}=1.58\right)$, inside the section of the river Matlapa. Between sub-basins, the greatest $\beta$ diversity was located in the river Huichihuayán $\left(H^{*}=2.36\right)$. Cichlasoma cyanoguttatum, Cichlasoma pantostictum, Cichlasoma steindachneri, Gambusia marshi, Ictalurus australis, Poecilia formosa and Poecilia reticulata were new records for the basin. Fish distribution was classified in six meta-basins. The most diverse family was Poeciliidae (14 species). Poecilia mexicana was the most abundant species followed by Astyanax mexicanus. Fifty one percent of the species were Neotropical, $20 \%$ Nearctic ones; $25.7 \%$ were native; only $2.8 \%$ were deliberately introduced. Chirostoma jordani and Notropis sallei were recent introductions from the Lerma river. Changes in habitat, channelization for agriculture, water transfers between rivers Lerma and Moctezuma, and the deliberate or accidental introduction and translocation of eight species, were the main reasons of the displacement and reduction in the distribution of the endemic species. Three of the recorded species are protected by Mexican environmental laws: Cichlasoma labridens, C. steindachneri and I. australis. Action plans to preserve fish community and the ecological processes at different territorial and time scales (meta-basin, basin and sub-basin) are proposed.
\end{abstract}

Key words: Biological conservation, ecosystemic model, ecology, biogeography, systematic.

\section{RESUMEN}

Diversidad biológica, distribución y estrategias de conservación de la ictiofauna de la cuenca del río Moctezuma, centro de México

La riqueza específica de la ictiofauna en la cuenca hidrográfica (ecoprovincia) del río Moctezuma fue de 35 especies y la $\gamma$ diversidad fue 2.5. En esta cuenca convergen dos regiones biogeográficas (Neártica y Neotropical) formando un corredor de transición entre regiones que favorece los endemismos por especiación vicariante. La $\alpha$ diversidad más alta se encontró en el ecotopo Jalpilla $\left(\mathrm{H}^{\prime}=1.58\right)$, dentro de la sección (ecosección) del río Matlapa. En una escala de subcuenca (ecodistrito), la $\beta$ diversidad más alta estuvo en Huichihuayán $\left(\mathrm{H}^{*}=2.36\right)$. Cichlasoma cyanoguttatum, Cichlasoma pantostictum, Cichlasoma steindachneri, Gambusia marshi, Ictalurus australis, Poecilia formosa y Poecilia reticulata son nuevos registros para la cuenca. Se clasificaron los patrones de distribución en seis metacuencas (ecorregiones). La familia mejor representada fue Poeciliidae (14 especies). Por su gran abundancia poblacional, destaca Poecilia mexicana seguida de Astyanax mexicanus. Dominan las especies neotropicales $51.5 \%$ frente al $20 \%$ de las neárticas; el $25.7 \%$ son nativas y solo el $2.8 \%$ son exóticas. Tanto Chirostoma jordani como Notropis sallei son de origen lermense y recientemente ingresaron al Moctezuma. Los cambios en el hábitat de las especies, la canalización de arroyos y ríos para la actividad agrícola y trasvases de agua del río Lerma al Moctezuma y viceversa, así como la introducción y translocación deliberada o accidental de ocho especies dulceacuícolas, son las causas principales del desplazo y reducción en la distribución de los endemismos. Tres especies están protegidas por la normatividad mexicana: Cichlasoma labridens, C. steindachneri $e$ I. australis. Se proponen planes de acción para conservar la ictiofauna y los procesos ecológicos de la cuenca a diferentes escalas de actuación.

Palabras clave: Conservación biológica, modelo ecosistémico, ecología, biogeografía, sistemática. 


\section{INTRODUCCIÓN}

La ictiofauna continental del centro de México se compone de 25 familias y 205 especies, de las cuales cerca del $63 \%$ son endémicas (Burr \& Mayden, 1992; Espinoza et al., 1993). Los problemas más graves a los que se enfrenta esta son el deterioro ambiental y la pérdida de sus hábitats naturales por factores antropogénicos (Alcocer \& Bernal, 2010; Gutiérrez-Yurrita \& López-Flores, 2011). Estos problemas se acentúan debido a una deficiente política de conservación y manejo de los recursos bióticos y a la creciente tendencia de introducir nuevas especies con fines comerciales (Gutiérrez-Yurrita, 2004); 11 especies de peces nativos han desaparecido por estas causas y por lo menos el $33 \%$ de peces dulceacuícolas (126 especies) se considera en riesgo de extinción (De la Vega-Salazar, 2006). La mayoría de la ictiofauna amenazada es endémica a ríos y manantiales con marcada estacionalidad de zonas (GutiérrezYurrita, 2012).
La cuenca del río Moctezuma es la más impactada por actividades humanas (trasvases, canalizaciones der ríos y entubamiento de manantiales, minería a cielo abierto que ha destruido casi totalmente las montañas, etc.) en México. Y es una de las más vulnerables a perder toda su riqueza biológica natural del país (GutiérrezYurrita 2011; De la Vega-Salazar, 2006).

En la cuenca Moctezuma el elevado número de endemismos de la ictiofauna ( $66 \%$ ) se debe en gran parte a su estratégica ubicación geográfica. Dos regiones biogeográficas confluyen en el área, la Neártica y la Neotropical; además de ser parte transicional de tres regiones fisiográficas mexicanas (Abell et al., 2000). A pesar de lo deteriorada ecológicamente que está la región en general, conserva amplias teselas paisajísticas con alto grado de conservación por ser poco accesibles al hombre (alta montaña con picos superiores a los $2500 \mathrm{msnm}$ cubiertos de bosque mixto de pino-encino y bosque de niebla); y profundos cañones a los que sólo se puede acceder al le-

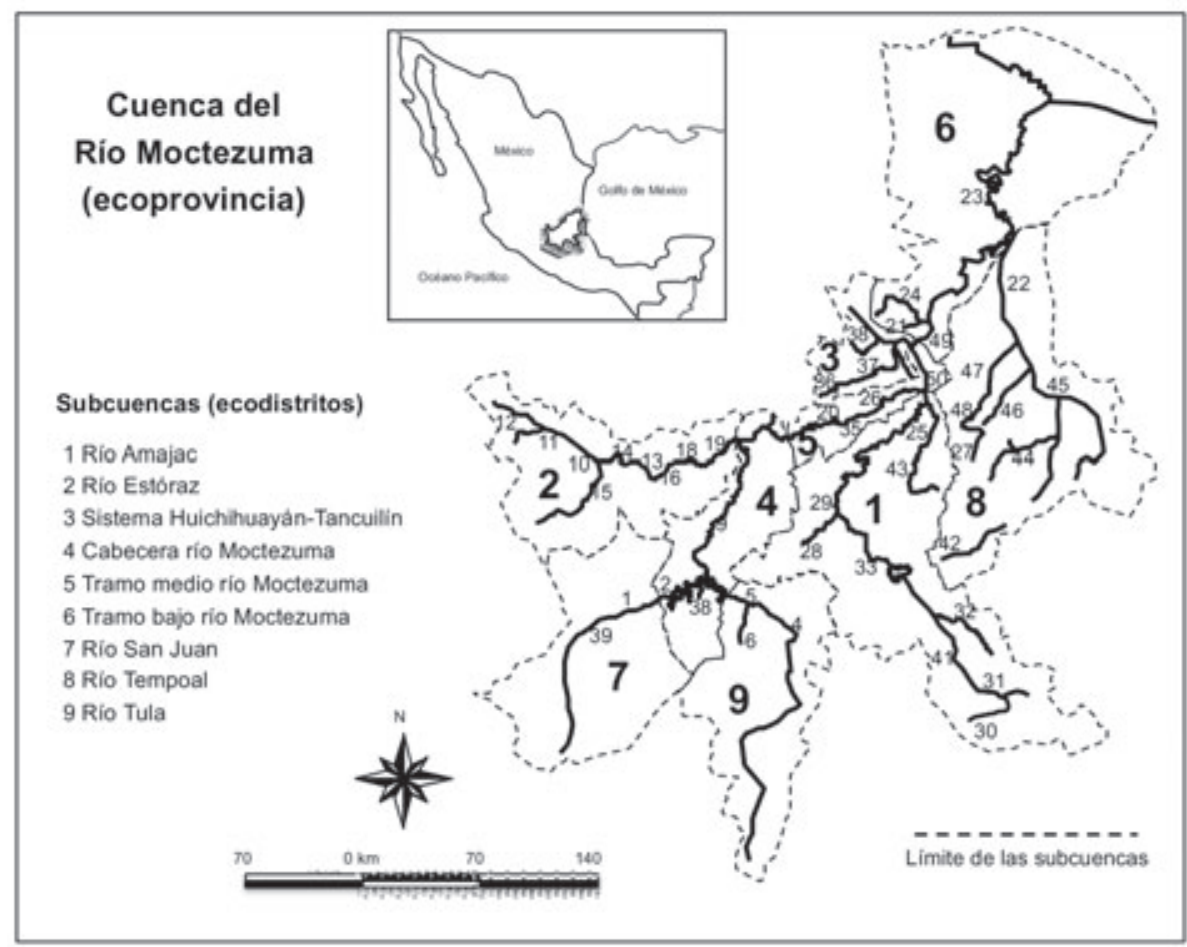

Figura 1. Localización del área de estudio con las nueve subcuencas que conforman la cuenca Moctezuma y los ecotopos de muestreo (el nombre de cada sitio de muestreo se encuentra en el Anexo 1: www.limnetica.net/internet). Study area with the nine sub-basins of the Moctezuma basin and the sampling ecotopes (the name of the sampling sites is in the Annex 1). 
cho del río por agua con lanchas especiales, cuya ribera está compuesta por selva baja caducifolia y selva alta perennifolia (Gutiérrez-Yurrita et al., 2002). Es importante señalar que dadas las condiciones meteorológicas y la abrupta orografía, la mayoría de los ríos que surcan la cuenca son altamente estacionales, perdiendo totalmente el caudal. Esta situación deja pozas y charcas temporales sin conexión unas con otras, que son utilizadas de manera intensiva por los pobladores locales como sitios de abrevadero de ganado y de extracción de agua para riego, principalmente (Gutiérrez-Yurrita 2012; Del Pozo et. al., 2012).

Bajo este panorama y dado el ritmo alarmante con el que se ha producido la extinción de especies y la destrucción de hábitats en la región (Gutiérrez-Yurrita et al., 2004), se ha incrementado la preocupación por los temas que permitan proteger y dotar de medidas de conservación determinadas áreas y especies ecológicamente importantes, como la elaboración de inventarios funcionales (Alonso-Eguía et al., 2002; Hurtado et al., 2005). Los inventarios funcionales sientan las bases de programas específicos de acción para conservar determinadas especies o procesos ecológicos indispensables para mantener la capacidad de resiliencia de los ecosistemas afectados. Por otro lado, sirven para actualizar las leyes de conservación en México (NOM-059SEMARNAT, 2010), y los listados internacionales de especies en riesgo, ya que toda actualización debe ir acompañada tanto de datos descriptivos y ecológicos de la especie a proteger, como de las estrategias que se proponen para asegurar la preservación y conservación de su hábitat.

El objetivo principal de este proyecto fue realizar un inventario funcional de la ictiofauna de la cuenca Moctezuma, partiendo de dos hipótesis: 1) La riqueza específica de la fauna endémica será mayor mientras menor sea el grado de alteración de la sección; 2) Los factores ambientales que controlan la distribución y abundancia de los peces en la cuenca del Moctezuma están subrogados entre sí dependiendo de la escala espacial y temporal a la cual se trabaje, de tal forma que pueden encontrarse los subrogados ecológicos para estimar la diversidad a diferentes niveles y proponer directrices de gestión de las sec- ciones y de la cuenca con un enfoque holístico (Gutiérrez-Yurrita et al., 2013).

\section{METODOLOGÍA}

\section{Área de estudio}

La cuenca Moctezuma abarca todas las subcuencas conectadas en red que conforman el sistema limnológico del río Moctezuma. Este complejo sistema se localiza en el centro de México y corre el flujo de agua hacia el occidente, desembocando en la gran cuenca del río Pánuco, formando así una ecozona (sistema de metacuencas conectadas) muy peculiar de México, dadas las contrastantes características geográficas, fisiográficas, geológicas, climáticas y orográficas que atraviesa (Fig. 1). La topografía espacial, cuyo gradiente altitudinal es muy amplio (75 a $2466 \mathrm{msnm}$ ), aunada a la división biogeográfica de México, como encrucijada entre las regiones Neártica y Neotropical, alberga grupos faunísticos distintivos por su origen. En la zona de estudio convergen tres ecorregiones dulceacuícolas de Norteamérica, "Cuenca del río Lerma", "Cabecera del Río Verde" y "Tamaulipas-Veracruz”, las cuales están situadas en la biorregión denominada "Zona de Transición Mexicana" (Abell et al., 2000). El resultado de esta biogeografía es un alto índice de endemismos.

\section{Muestreo}

La cuenca Moctezuma (ecoprovincia) se dividió en nueve subcuencas (ecodistritos) y cada una de ellas en tres tramos, siguiendo el criterio de considerar un río como un continuo (Vannote et al., 1980) (Fig. 1). Se realizaron muestreos en las secciones donde había algo de información sobre la biota y ecología del sitio; se visitaron los ecotopos que teóricamente estaban mejor muestreados, para evaluar cómo habían cambiado las comunidades de peces a lo largo del tiempo y tratar de dilucidar las causas del cambio. El lugar específico de colecta se consideró como el ecotopo (hábitat) de las Unidades Taxonómicas Operativas presentes. 
El estudio duró 18 meses con muestreos quincenales en 50 ecotopos (Fig. 1) que comprenden embalses, lagos, manantiales, arroyos y ríos de la cuenca Moctezuma.

\section{Clasificación y taxonomía de la ictiofauna}

El material ictiológico se colectó utilizando diferentes sistemas de muestreo específicos para cada sistema acuático (red de arrastre de $30 \mathrm{~m}$ por $1.5 \mathrm{~m}$ de tiro, con una luz de malla de $5 \mathrm{~mm}$, redes de cuchara con diferente luz de malla y equipo de electro pesca). Cada lote de peces está asociado a una base de datos con diferentes campos, referida en el formato determinado por el programa de cómputo BIÓTICA, desarrollado ex profeso por la Comisión Nacional para el Conocimiento y Uso de la Biodiversidad (CONABIO).

La clasificación taxonómica se realizó consultando las claves y los criterios de Álvarez (1970), Arredondo-Figueroa \& Guzmán-Arroyo (1985), Burr \& Maiden (1992), Castro-Aguirre et al. (1999), De Buen (1947a, b, c), Espinosa et al. (1993), Miller (1986), Miller \& Smith (1986), Schultz \& Miller (1971), Taylor \& Miller (1983); además, se consultaron diversos textos históricos y a expertos en ictiología de la Escuela Nacional de Ciencias Biológicas del IPN, el Instituto de Biología de la UNAM y de la UAM-Iztapalapa, para corroborar ciertas determinaciones y ver los ejemplares tipo de algunas de las especies.

\section{Índices de diversidad biológica $(\alpha, \beta, y)$}

La diversidad biológica denominada como diversidad alfa $(\alpha)$ se estimó con el índice de diversidad de Shannon $\left(H^{\prime}\right)$, y la diversidad gamma $(\gamma)$ para evaluar la diferenciación de los paisajes (Magurran 1989). La diversidad beta $(\beta)$ se usó para estimar la heterogeneidad paisajística mediante el recambio de especies en el espacio, bajo dos criterios: el de Whittaker y el de Jaccard $(\mathrm{Sj})$. De esta forma, se generó un dendograma para comparar las hipótesis de similitud y recambio de especies en una asociación biológica (gradual y discontinua). Se utilizó el índice de Shannon $\left(H^{\prime}\right)$ como medida de diversidad acumulada $\left(H^{*}\right)$, y la diferencia de este valor con el de cada hábitat reveló la tasa de recambio de especies de ese hábitat (Whittaker, 1975). El procedimiento Jaccard $(\mathrm{Sj})$ se empleó en contraposición al método de las discontinuidades por el tipo de información recabada (presencia-ausencia de UTO's) y por ser estadísticamente más robusta la estimación de la similitud de hábitat que el simple método de Bray-Curtis (Magurran, 1989). La comparación estadística entre los índices Jaccard y Bay-Curtis se realizó mediante un análisis de escalamiento multidimensional usando el paquete estadístico PAST (Hammer et al., 2001). Finalmente, bajo los mismos criterios, se entiende que la diversidad $\gamma$ es resultado de la combinación de dos niveles de diversidad, por un lado la diversidad $\alpha$ y por otro la diversidad $\beta$ (AlonsoEguía et al., 2002). Para asignar confianza estadística a estos índices, se utilizó un análisis multivariante de agrupación jerárquica entre los sitios de muestreo y las subcuencas del río Moctezuma (McMarigal et al., 2000).

\section{Distribución estadística y dispersión ecológica}

Como primera medida de aproximación a la dispersión natural de las poblaciones de peces, se estimó su distribución en gradientes altitudinales. Para ello se definieron cinco intervalos con una diferencia de $500 \mathrm{~m}$ de altitud cada uno. A partir de este análisis grueso, se afinaron las distribuciones con datos de altura cada $100 \mathrm{~m}$ e introdujeron los factores fisicoquímicos más relevantes en la ictiofauna, usando un equipo Horiba U-23 water-chequer con dataloger acoplado para agregar los datos en la computadora: conductividad, porcentaje de salinidad, oxígeno disuelto, $\mathrm{pH}$ y temperatura; para estandarizar la varianza entre variables mencionadas. Se construyó una tabla de frecuencia absoluta de ocurrencia con intervalos de distribución de cada especie por factor analizado, previamente estandarizado estadísticamente. Para un respaldo estadístico del análisis tanto de altitud como de factores fisicoquímicos, se utilizó un análisis de ordenación por correspondencia (DECORANA) con el programa Minitab Release 15-2006, donde $\chi^{2}$ mide el grado de asociación entre los factores (intervalos estandarizados de los parámetros fisi- 
coquímicos) y las UTO's (presencia-ausencia de especies en cada intervalo), en una tabla de contingencia con I renglones (especies) y J columnas (intervalos de los factores fisicoquímicos); de esta forma, se llevó el índice de diversidad biológica a una cuantificación escalar, pasando de diversidad $\alpha$ a diversidad $\beta$ (Whittaker, 1975).

\section{RESULTADOS}

Se determinaron taxonómicamente 3420 individuos. Estos ejemplares se asignaron a 35 UTO's, las cuales pertenecen a 11 familias y 20 géneros. Siete especies corresponden a nuevos registros para la cuenca y tres están amenazadas de extin-

Tabla 1. Elenco ictiofaunístico de la cuenca hidrográfica (ecoprovincia) del Moctezuma. Aut: Autóctona; Ex: Exótica; En: Endémica; Trans: Translocada; A: Amenazada R: Rara; Pr: Sujeta a Protección especial; 1: Amajac; 2: Estóraz; 3: Huichihuayán; 4: Cabecera del Moctezuma; 5: Moctezuma tramo medio; 6: Moctezuma tramo bajo; 7: San Juan; 8: Tempoal; 9: Tula.* Indica que esta especie es nuevo registro para la cuenca. Fish inventory of the Moctezuma basin (ecoprovince). Aut: Autoctonous; Ex: Exotic; En: Endemism; Trans: Translocated; A: Endangered; R: Rare; Pr: special protection. * This species is a new record to the basin.

\begin{tabular}{|c|c|c|c|}
\hline Familia & Especie & Estatus & Subcuenca \\
\hline Atherinopsidae & Chirostoma jordani (Woolman, 1894) & Aut & 1 \\
\hline Characidae & Astyanax mexicanus (De Filippi, 1853) & Aut & $1 ; 2 ; 7 ; 9 ; 4$ \\
\hline \multirow[t]{3}{*}{ Catastomidae } & Ictiobus labiosus (Meek, 1904) & Aut & 5 \\
\hline & Carassius auratus (Linnaeus, 1758) & Ex & $1 ; 9$ \\
\hline & Cyprinus carpio (Linnaeus, 1758) & Ex & $1 ; 9$ \\
\hline \multirow[t]{10}{*}{ Cyprinidae } & Dionda ipni (Álvarez y Navarro, 1953) & Aut & $1 ; 35$ \\
\hline & Notropis cf. chihuahua (Woolman, 1982) & Aut & 5 \\
\hline & Notropis cf. saladonis (Hubbs y Hubbs, 1958) & Aut & $4 ; 8$ \\
\hline & Notropis sallei (Günther, 1968) & Aut & 2 \\
\hline & Gambusia atrora (Rosen y Bailey, 1963) & Aut & $1 ; 3 ; 6$ \\
\hline & Gambusia marshi (Minckley \& Craddock, 1962) & Aut $*$ & 6 \\
\hline & Gambusia vittata (Hubbs, 1926) & Aut & 6 \\
\hline & Heterandria bimaculata $($ Heckel, 1848) & Aut & $4 ; 7 ; 9$ \\
\hline & Heterandría jonesii (Günther, 1874) & Aut & $1 ; 3 ; 8$ \\
\hline & Poecilia formosa (Girard, 1859) & Aut & 6 \\
\hline \multirow[t]{8}{*}{ Poeciliidae } & Poecilia mexicana (Steindachner, 1863) & Aut & $1-9$ \\
\hline & Poecilia reticulata (Peters, 1860) & $\mathrm{Ex} *$ & 5 \\
\hline & Poecilipsis gracilis (Heckel, 1848) & Aut & $6-9$ \\
\hline & Xiphophorus cortezi (Rosen, 1960) & Aut & $1 ; 3$ \\
\hline & Xiphophorus. hellerii (Heckel, 1848) & Ex & 1 \\
\hline & Xiphophorus malinche (Rauchen, Kallman \& Mozirot, 1990) & Aut & 8 \\
\hline & Xiphophorus birchmanni (Lenchner \& Radda, 1987) & Aut & $1 ; 6 ; 8$ \\
\hline & Xiphophorus pymaeus (Hubbs \& Gordon, 1943) & Aut & 3 \\
\hline Goodeidae & Goodea gracilis (Hubbs \& Turner, 1939) & En & $2 ; 4 ; 7 ; 9$ \\
\hline \multirow[t]{3}{*}{ Mugilidae } & Agonostomus monticola (Bancroft, 1834) & Aut & 3 \\
\hline & Cichlasoma cyanoguttatum (Baird \& Girard 1854) & Aut; Trans* & $2 ; 4 ; 7 ; 9$ \\
\hline & Cichlasoma labridens (Pellegrin, 1903) & Aut; A * & $1 ; 3 ; 5 ; 8$ \\
\hline \multirow{4}{*}{ Cichlidae } & Cichlasoma steindachneri (Jordan \& Snyder, 1899) & Aut; R; Pr* & $1 ; 3 ; 5 ; 8$ \\
\hline & Cichlasoma pantostictum (Teylor \& Miller, 1989) & Aut* & $1 ; 3 ; 5 ; 8$ \\
\hline & Oreochromis aureus (Steindachner, 1864) & Ex & $1 ; 2 ; 4 ; 7-9$ \\
\hline & Oreochromis mossambicus (Peters, 1852) & Ex & 1 \\
\hline \multirow{2}{*}{ Centrarchidae } & Lepomis macrochirus (Rafinesque, 1819) & Ex & $7 ; 9$ \\
\hline & Micropterus salmoides (Lacepède, 1802) & Ex & 7 \\
\hline Eleotridae & Gobiomorus dormitor (Lacepède, 1800) & Aut & $5 ; 3$ \\
\hline Ictaluridae & Ictalurus australis (Meek, 1904) & Aut; A & 5 \\
\hline
\end{tabular}


ción y protegidas por la NOM-059-ECOL-2001 (Tabla 1). De estas especies, 12 son primarias (estrictamente dulceacuícola), 21 secundarias (tolerantes a aguas salinas) y dos son derivadas del medio marino: una catádroma (A. monticola) y otra eurihalina $(G$. dormitor). Se encontraron 26 especies nativas y nueve exóticas.

La familia mejor representada es Poeciliidae, con 14 especies, seguida de Cichlidae y Cyprinidae con 6 especies cada una; Centrarchidae presenta 2 especies. Siete familias están representadas con 1 especie.

En cuanto al origen biogeográfico, dominan las especies neotropicales, 18 especies $(51.5 \%)$ frente a siete de origen neártico (20\%). El 8.5\% de especies son de origen marino que remontan ríos y el $20 \%$ restante son introducidas o translocadas.

En la distribución de la ictiofauna destaca $P$. mexicana, que se colectó en 29 de los 50 sitios muestreados; A. mexicanus es la segunda especie en abundancia, presentándose en 14 sitios, seguida de $C$. cyanoguttatum y $O$. aureus, colectadas en 12 sitios; A. monticola, C. auratus, N. cf. saladonis, N. sallei, Ch. jordani, O. mossambicus, I. australis, G. atrora, P. reticulata, X. malinche, X. hellerii, y X. pygmaeus, se colectaron en un solo sitio.

En cuanto a la riqueza específica, los ecotopos con mayor número de especies (8 especies) fueron el río Claro (sección localizada en San Luis Potosí), Laguna Metztitlán (sección en Hidalgo) y río Candelaria (sección en Hidalgo). En lo que se refiere a la riqueza específica por subcuenca, la del Amajac presenta el mayor nú- mero de especies (18), seguida de Huichihuayan con 16 y Tempoal con 15 .

El índice de diversidad $\alpha$ más elevado se encontró en el ecotopo Jalpilla, ubicado en la sección río Matlapa $\left(H^{\prime}=1.58\right)$; seguido del arroyo San Andrés y río Claro, con valores superiores a 1.3 (Anexo I, material suplementario consultable en www.limnetica.net/internet). El índice de diversidad biológica $\beta$ para cada subcuenca por el método de Whittaker $\left(H^{*}\right)$ estuvo en un intervalo de 1.22 a 2.36 (Tabla 2). El índice de diversidad $\gamma$ para toda la cuenca tuvo el valor de $H^{* *}=2.5$.

Con el análisis de Jaccard se encontraron siete asociaciones biológicas que comparten al menos el $50 \%$ de la ictiofauna, a nivel de cuenca (Fig. 2); mientras que a escala de subcuenca se localizaron tres asociaciones (Fig. 3). En la Tabla 3 se muestran las asociaciones ícticas de la zonificación de la cuenca. Los manantiales que ya no tenían conexión con los sistemas acuáticos mayores tuvieron cero especies en común con el resto de las secciones y cuenca. El análisis comparativo entre los dos índices de diversidad $\beta$ no mostró diferencias significativas (Fig. 4). Los ecotopos Río Vando los Martínez (8), manantial en Vando los Martínez (9) y el río Tultitlán (27) sobresalen como vértices antagónicos de los puntos del polígono que encierra la mínima área de la dispersión de datos.

La afinidad biológica ictiofaunística entre los sitios muestreados permitió determinar tipos de subcuencas que de manera operativa quedan así: 1) subcuencas San Juan, Tula y Cabecera del río

Tabla 2. Índices de diversidad $\beta$ estimados como ponderación de los índices de Shannon $\left(H^{\prime}\right)$, para cada una de las subcuencas (ecodistritos) de la cuenca del Río Moctezuma. $\beta$ diversity taking into account the Shannon $\left(\mathrm{H}^{\prime}\right)$ indices for every sub-basin (ecodistricts) in the Moctezuma basin.

\begin{tabular}{lccc}
\hline Subcuenca & No. especies & No. Ejemplares & $H^{*}$ \\
\hline Huichihuayán & 16 & 335 & 2.36 \\
Amajac & 18 & 839 & 1.85 \\
Extorás & 5 & 248 & 1.28 \\
Tempoal & 15 & 798 & 1.74 \\
Moctezuma tramo medio & 10 & 316 & 1.59 \\
Moctezuma tramo bajo & 7 & 55 & 1.22 \\
San Juan & 5 & 237 & 1.22 \\
Cabecera del Moctezuma & 7 & 377 & 0.88 \\
Tula & 8 & 218 & 0.69 \\
\hline
\end{tabular}




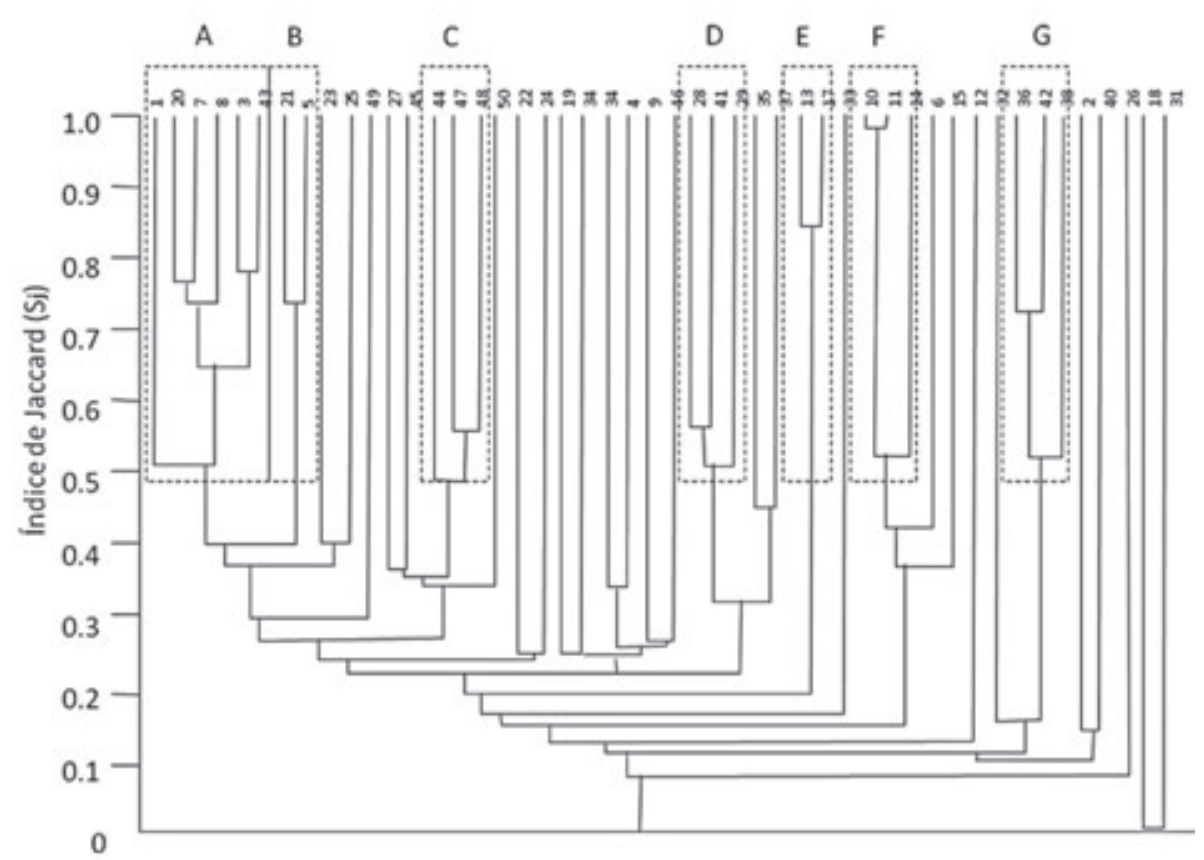

Figura 2. Comparación métrica de los coeficientes de similitud de la ictiofauna de todos los sitios de colecta de los ecotopos. La agrupación se realizó con valores de 0.5 a 1.0. Metric comparison of the similarity coefficients from fish community at the sampling sites. Clusters were performed with values from 0.5 to 1.0.

Moctezuma, con variedad de especies primarias; 2) subcuenca del río Estóraz con predominio de G. gracilis, A. mexicanus y N. sallei; 3) subcuencas del Tempoal y Huichihuayan con mayor ocurrencia de especies secundarias.

Del total de especies, 25 (el 71\%) se presentan en la parte más baja de la cuenca entre 75 y $500 \mathrm{msnm}$. Sobresalen por ser endémicas a esta área: G. dormitor, $N$. cf. chihuahua, $N$. cf. saladonis, C. pantostictum, C. steindachneri, G. atrora, G. marshi, G. vittata, P. formosa, P. reticulata, X. birchmanni, X. malinche, X. pygmaeus e I. labiosus. El intervalo altitudinal que resultó con menor número de especies corresponde a la parte más alta de la ecozona (2000 a $2500 \mathrm{msnm}$ ), con el $17 \%$ de las especies determinadas para la cuenca; tres UTO's de esta ecozona son introducidas. En cuanto a las especies con el mayor intervalo de distribución, P. mexicana destaca por tener un intervalo que va de los 75 a $2466 \mathrm{msnm}$; seguida de A. mexicanus de 75 a $1400 \mathrm{msnm}$; y de G. gracilis de 900 a 2000 msnm.

La clasificación del área de estudio por dispersión altitudinal de la ictiofauna, con resolución de

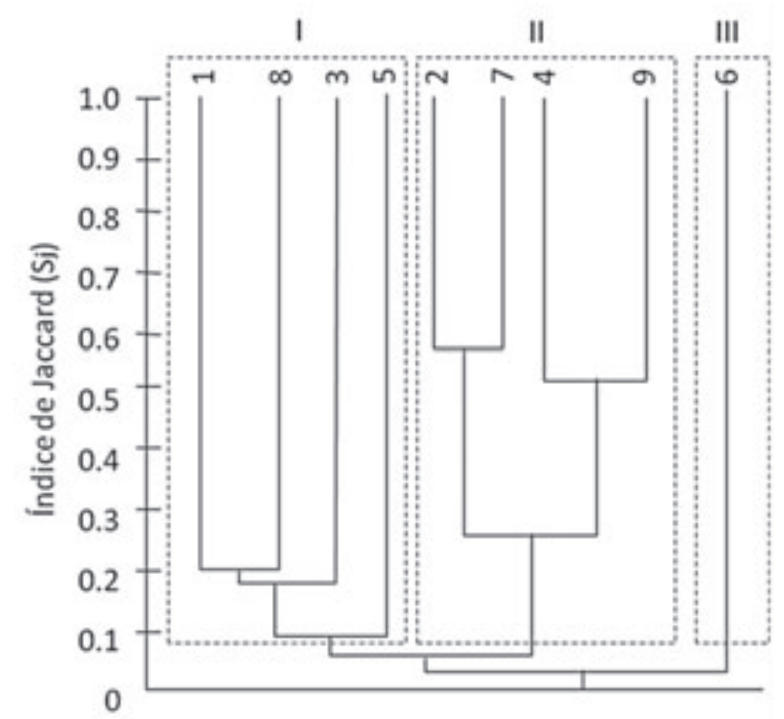

Figura 3. Comparación métrica de los coeficientes de similitud de los ecotopos que resultaron sobresalientes en cada una de las subcuencas en las que se subdividió la cuenca del río Moctezuma (el significado de los números romanos en la Tabla 2). Metric comparison of the similarity coefficients of the ecotopes distinguished at each sub-basin that the Moctezuma basin was subdivided (the meaning of the Roman numbers are shown in Table 2). 
curvas de nivel cada $500 \mathrm{~m}$ y dicha de manera operativa es como sigue: 1) subcuencas de los ríos San Juan, cabecera del río Moctezuma, Tula y Estóraz, las cuales comprenden la distribución de G. gracilis en toda la cuenca. En este grupo se reconocen asociaciones de peces exclusivos para la cabecera del río Moctezuma con altitudes mayores a 900 msnm y comunidades de peces exclusivas de la subcuenca del Estóraz; 2) subcuencas del tramo medio del río Tempoal. Incluye las aportaciones de los ríos Huichihuayan y Amajac. Su variación altitudinal está entre 250 y 900 msnm; 3) subcuencas del tramo bajo del río Tempoal, con una variación en su altitud de 75 a 250 msnm.

El análisis altitudinal a intervalos de $100 \mathrm{~m}$ muestra seis patrones de distribución, con excepción de $P$. mexicana, que se localiza en toda la cuenca (Fig. 5: 1) especies exclusivas de la parte media, ubicadas al poniente de la cuenca del Moctezuma (subcuencas de los ríos San Juan del Río, Estóraz y cabecera del Moctezuma); N. sallei y M. salmoides; 2) especies exclusivas de la parte alta: C. auratus, L. macrochirus, M. salmoides, H. bimaculata y Ch. jordani; 3) especies exclusivas de la parte media, al oriente de la cuenca (subcuenca Amajac); X. hellerii, C. carpio y $O$. mossambicus; 4) especies que se presentan en un intervalo de dispersión mayor a $1400 \mathrm{msnm}$, y que se distribuyen al poniente, en parte alta y media de la cuenca ( $H$. bimaculata y G. gracilis); 5) especies que no rebasan los $1400 \mathrm{msnm}$, las cuales se distribuyen en la parte media y baja de la cuenca (H. jonesii, X. cortezi, C. labridens y D. ipni.; 6) especies exclusivas de la parte baja: G. dormitor, N. cf. chihuahua, N. cf. saladonis, C. pantostictum, C. steindachneri, G. atrora, G. marshi, G. vittata, P. formosa, P. reticulata, $X$.

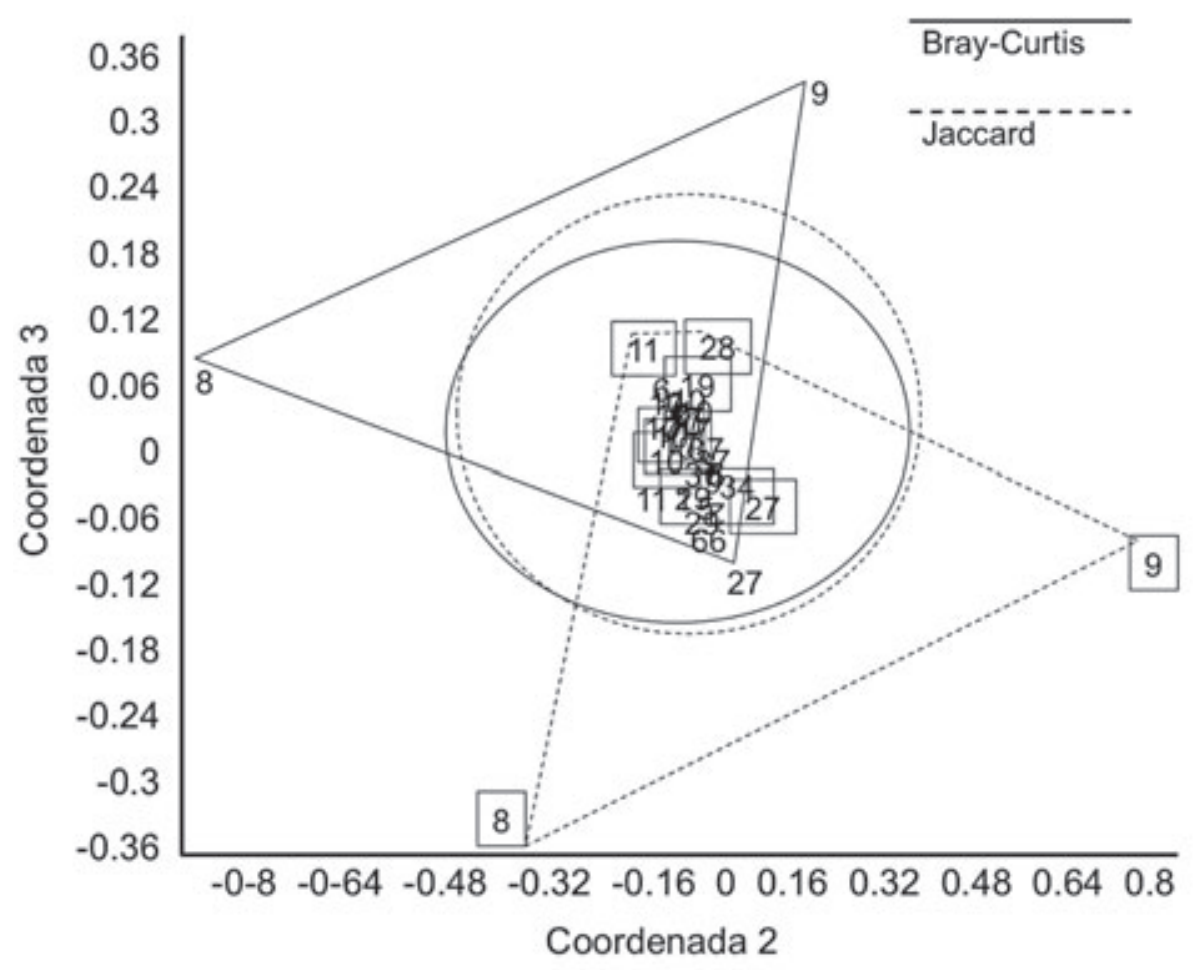

Figura 4. Diagrama de ordenación escalar multidimensional no métrica con los valores de los índices de disimilitud (Bray-Curtis) y de similitud (Jaccard) para cada ecotopo de la cuenca Moctezuma. El polígono representa el área mínima de la dispersión de los datos con el método Minimum Span Convex Hull; las elipses presentan el nivel de confianza al $95 \%$. Non metric multidimensional scaling ordination using the values of the indices of dissimilarity (Bray-Curtis) and similarity (Jaccard) for each ecotope of the Moctezuma basin. The polygon represents the minimal area of the data dispersion with the method Minimum Span Convex Hull; the ellipses represent the confidence level at $95 \%$. 
birchmanni, X. malinche, X. pygmaeus, I. labiosus, A. monticola e I. australis.

La dispersión de la ictiofauna respecto a los factores físico-químicos del agua se puede sintetizar así: 1) Conductividad: $17 \%$ de las especies se distribuyen en un intervalo 0.223 a $1.99 \mathrm{mS} / \mathrm{cm} ; 37 \%$ de la ictiofauna se colectó en intervalos de 0.223 a $0.51 \mathrm{mS} / \mathrm{cm} ; 8 \%$ se presentó en intervalos de 1.03 a $1.99 \mathrm{mS} / \mathrm{cm}$; G. gracilis es la única especie colectada en el intervalo de 0.0 a $1.99 \mathrm{mS} / \mathrm{cm}$. 2) pH: $31 \%$ de las especies presentan el mayor intervalo que va de 6.1 a 9.4; dos especies se dispersan en intervalos de 6.1 a 7.0. 3) Salinidad: $17 \%$ de las especies fueron colectadas entre 0.0 y $0.1 \mathrm{ppm} ; 5 \%$ se colectaron en sitios con baja concentración (0.0$0.009 \mathrm{ppm}$ ). 4) Oxígeno disuelto: $42 \%$ de las especies fueron colectadas entre 1.52 y $15 \mathrm{mg}$
$\mathrm{L}^{-1} ; 11 \%$ se colectaron en cuerpos de agua con un intervalo de 9.2 a $15 \mathrm{mg} \mathrm{L}^{-1}$. 5) Temperatura: 3 especies se localizaron entre los 15.1 y los $32.4^{\circ} \mathrm{C} ; 7$ especies se localizaron en el intervalo de 15.1 a $27.3^{\circ} \mathrm{C} ; 16$ especies $(46 \%)$ se colectaron en el intervalo de 19.2 a $23.2^{\circ} \mathrm{C}$.

\section{DISCUSIÓN}

La ictiofauna de la cuenca del río Moctezuma, como cuenca, incluye taxa de origen Neotropical (sudamericano) y Neártico (norteamericano); A su vez, presenta un alto porcentaje de poblaciones de especies biológicas (UTO's) autóctonas. Las UTO's de esta cuenca se distribuyen de manera gradual y discreta a lo largo de la misma cuenca, puesto que los factores ambientales que

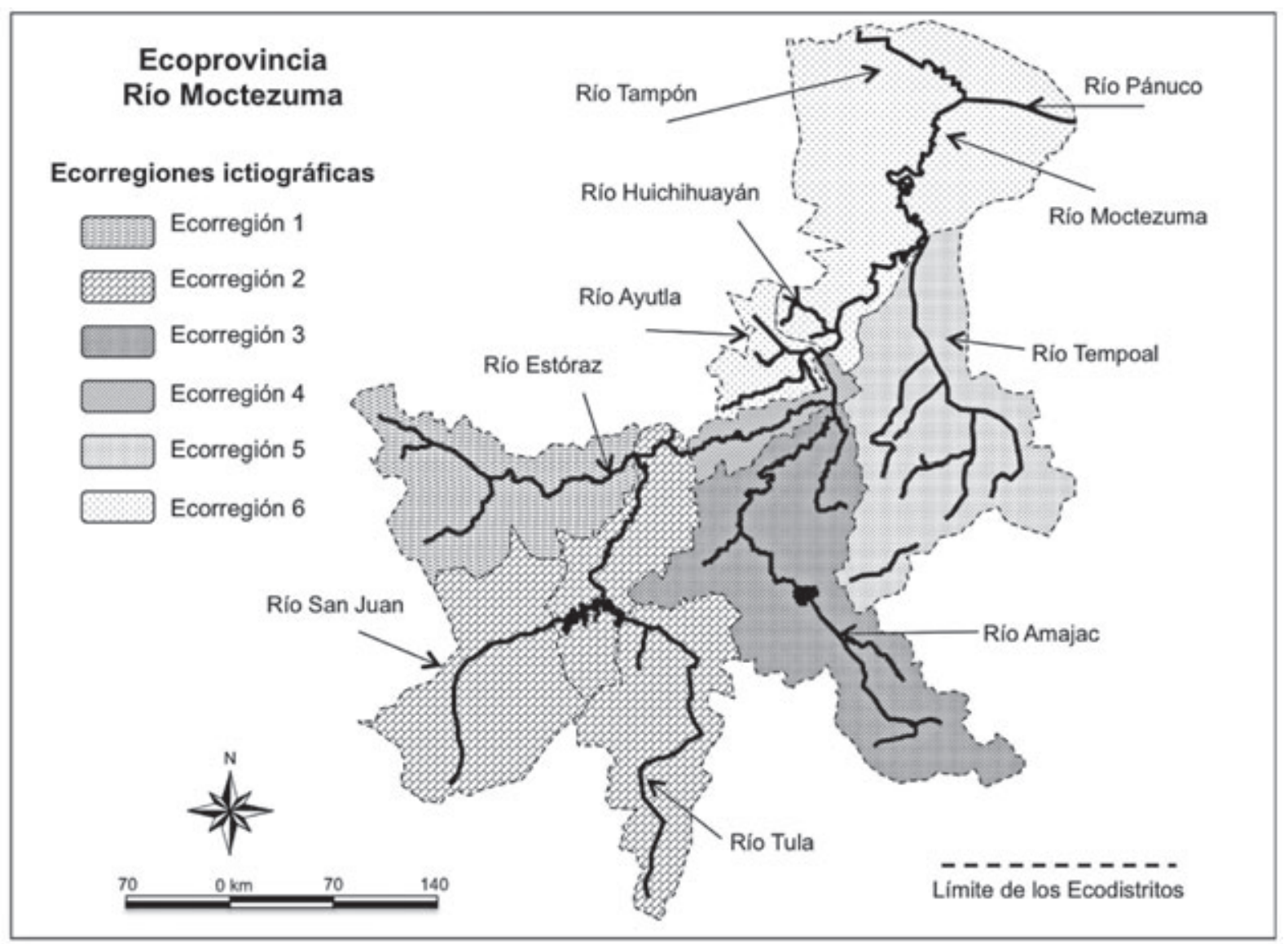

Figura 5. Zonificación de la distribución de la ictiofauna de la cuenca Moctezuma con fines de conservación de sus poblaciones. Fish distribution zonation in the Moctezuma basin for conservation purposes. 
controlan la comunidad de peces están correlacionados y subrogados entre las escalas regionales y locales (Gutiérrez-Yurrita \& Morales-Ortiz, 2002). De esta forma, en un contexto fisiográfico, se conjugan las hipótesis de un continuo y de discontinuidades intra e inter secciones, pilares del Sistema Nacional de Áreas Naturales Protegidas (SiNANP) de México, cuya meta es contar con un sistema de ANP donde se aseguren los procesos de conectividad y permeabilidad ecológica. El sistema de gestión de la SiNANP está basado en el holismo, de tal forma que se vinculen las actividades humanas a las funciones ecológicas de las meta-cuencas conectadas en red (GutiérrezYurrita et al., 2013).

Por lo que se refiere a los peces, la distribución de las familias Cichlidae y Poeciliidae, de origen neotropical, puede tomarse como un índice claro del límite entre las dos áreas biogeográficas (Álvarez, 1972). Asimismo, la vertiente del Atlántico de la provincia ictiofaunística de la Meseta Central de México se ha marcado como una zona de transición entre estas dos regiones ( $\mathrm{Mi}$ ller, 1982). Las cuencas Moctezuma y Pánuco, englobadas en una ecozona formada por el sistema limnológico Moctezuma-Pánuco, funciona como corredor ecológico entre las áreas biogeográficas neártica y neotropical, propiciando el alto porcentaje de especies de origen neotropical $(51.5 \%)$ encontradas, respecto al de origen neártico $(20 \%)$. Se establece así el amplio predominio de la familia Poeciliidae (14 especies). Miller (1982) propuso que el origen de los peces neotropicales en esta región se debe a que estos remontaron ríos desde el Atlántico.

Algunas especies representadas en esta cuenca sirven para dar testimonio de la importancia biológica de la zona Mesoamericana y de la conectividad ecológica entre cuencas, las cuales funcionarían como metacuencas. El continuo y actual trasiego de fauna Lermense (sistema limnológico que drena hacia el Océano Pacífico) hacia el Moctezuma-Pánuco hace suponer que todavía la capacidad de carga de los ecosistemas del Moctezuma no está en su máximo. Para ilustrar el caso, se tiene que Ch. Jordani y $N$. sallei recientemente se integraron a la cuenca del Pánuco; mientras que para el género difilético Goodea con distribución en ambas cuencas se sugiere una colonización no tan reciente que pasó por un periodo de aislamiento, encontrando a G. gracilis solamente en la cuenca del Pánuco y $G$. atripinis en la cuenca del Lerma. Ambos casos se han explicado en el sentido de que las especies colonizadoras tienen poco solapamiento de nicho (Odling-Smee et al., 2003) por estar filogenéticamente distanciadas; estos ambientes servirán, entonces, como nodos funcionales de transferencia de energía (Gutiérrez-Yurrita \& Montes, 1999; Chase \& Leibold, 2003).

La importancia de enfocar el estudio bajo el paradigma ecosistémico y realizar la escala espacial y temporal en cuencas y subcuencas como escalas operativas (Fig. 5) es que para el manejo de la diversidad de la ecozona Moctezuma-Pánuco puede emerger una región ecológica nueva a una escala de ecorregión, la metacuenca. Las metacuencas, por definición, son cuencas que conservan su conectividad ecológica mediante un efectivo flujo genético y de energía, en una forma similar, aunque no igual, al de las ya bien estudiadas metapoblaciones (Raventós et al., 2005).

El modelo de funcionamiento de la cuenca que se propone la divide en seis ecorregiones bien definidas (Fig. 5), en donde algunas subcuencas

Tabla 3. Taxones asociados por el agrupamiento de las subcuencas que conforman la cuenca del río Moctezuma a partir del análisis de agrupamiento. Fish associated taxa to sub-basins clustering in the Moctezuma basin.

\begin{tabular}{|c|c|c|}
\hline Grupo & Subcuencas & Asociación ecológica de especies \\
\hline $\mathrm{I}$ & $\begin{array}{l}\text { Extoras, Cabecera del Moctezuma, San Juan } \\
\text { y Tula. }\end{array}$ & $\begin{array}{l}\text { A. mexicanus, C. auratus, C. carpio, } N \text {. sallei, C. cyanoguttatum, O. aureus, } \\
\text { L. machrochirus, M. salmoides, H. bimaculata, P. mexicana y G. gracilis. }\end{array}$ \\
\hline II & $\begin{array}{l}\text { Amajac, Huichihuayán, Moctezuma tramo } \\
\text { medio y Tempoal. }\end{array}$ & $\begin{array}{l}\text { A. montícola, A. mexicanus, C. carpio, } N . \text { cf. Chihuahua, N. cf. saladonis, } \\
\text { C. jordani, G. dormitor, C. labridens, C. pactostictum, C. steindachneri y } \\
\text { O. mossambicus. }\end{array}$ \\
\hline III & $\begin{array}{l}\text { Moctezuma tramo bajo (hasta su } \\
\text { desembocadura en el río Pánuco). }\end{array}$ & $\begin{array}{l}\text { A. mexicanus, N. cf. chihuahua, C. labridens, G. marshi, G. vittata, P. Formosa } \\
\text { у P. mexicana. }\end{array}$ \\
\hline
\end{tabular}


como la del Huichihuayán y la del Amajac funcionan como semilleros de individuos, dada su madurez ecológica y geológica; otros distritos más jóvenes geológicamente hablando como el San Juan y el Estóraz son receptores. En este sentido, las secciones de las subcuencas del río Moctezuma (cabecera, tramo medio y tramo bajo) funcionan como corredores de la ictiofauna, ya que son los puntos de unión entre las demás subcuencas para conformar la ecorregión o metacuenca, que une el río Moctezuma con el río Pánuco. Sin embargo, la velocidad de cambio y/o colonización de UTO's, dependerá de la fracción de ecotopos ocupados. Cuando se acaba de formar un ecotopo la proporción de espacios ocupados es cero y un ecotopo maduro tiene una fracción igual o cercana a 1 (Raventós et al., 2005).

Por otro lado, hay que resaltar cuatro causas explicativas por las cuales algunas especies registradas en este estudio, conspicuas y relativamente abundantes, no hayan sido registradas o colectadas anteriormente: 1) malas determinaciones taxonómicas en los trabajos anteriores; 2) cambios importantes en el hábitat, propiciando que se modifique la distribución y abundancia numérica de muchas especies; 3) aparición de especies exóticas, algunas introducidas deliberadamente con fines de pesca como la competitiva tilapia (O. mossambicus) o la gran depredadora lobina (M. salmoides), y otras translocadas, tal vez con la misma finalidad, como la mojarra nativa del bajo Pánuco C. cyanoguttatum; y 4) carencia de muestreos sistemáticos, dejando grandes vacíos de información en la cuenca. En este último punto sería muy conveniente utilizar un método más sistemático para detectar dónde se han colectado ciertas especies y dónde hay un número bajo de las mismas, a través de curvas de saturación de especies, por ejemplo, y con esa información, reforzar los estudios en la regiones donde haya vacíos (Alonso et al., 2002; Gutiérrez-Yurrita et al., 2004; Bruno et al., 2012).

Los valores de diversidad $\alpha$ para cada sección son comparativamente bajos (valores inferiores a $H^{\prime}=2$ y muchos menores que uno), incrementándose de forma gradual conforme disminuye la altitud. Aunque los valores de la diversidad $\beta$ entre las subcuencas son relativamente bajos, con excepción de Huichihuayán $\left(H^{*}=2.36\right)$, se incrementan hasta llegar a un valor máximo para la cuenca de diversidad $\gamma\left(H^{* *}=2.5\right)$. Una de las razones por las cuales se usó la función de $\mathrm{H}$ como índice de diversidad $\beta$, fue porque, al ser acumulativa, incrementa el valor conforme se incrementa la escala medición. Este incremento sirve para realizar una diferencia con los valores de diversidad $\alpha$ y obtener la estimación de cambio cuantitativo de especies y su abundancia de cada sección respecto a su subcuenca. Cuando el hábitat es homogéneo y el cambio de especies gradual, la diversidad $\beta$ no es estadísticamente superior a la diversidad $\alpha$ (Alonso et al., 2002), como ocurre en este último caso con la subcuenca Huichihuayán, que es un río con flujo lento, sin cambios aparentes de altitud y una estructura vegetal muy homogénea y uniforme en toda la rivera.

En general, en las ecorregiones donde el gradiente altitudinal es más pronunciado, mayor es la diferencia o discontinuidad de las comunidades ícticas, no solo porque hay más cambios bruscos en al ambiente físico, sino porque los límites de tolerancia de las especies se vuelven más definidos a causa de la competencia y los procesos coevolutivos entre los taxa interactuantes e interdependientes (Tilman, 1994). Los bajos índices de similitud de Jaccard, entre secciones, son resultado no sólo de la heterogeneidad de asociaciones de especies, sino que también son el reflejo de la variedad de hábitats muestreados.

Los ambientes regulados por el hombre, más en concreto, la parte alta de la cuenca (presa Zimapán, río Alfajayucan, río Tula, río San Juan), presentan bajos valores de diversidad por la homogeneización de los hábitats producidos por el represamiento (Holumuzki, 2000; Hurtado et al., 2005). En este sentido, Power et al. (1988) mencionan que dentro de los controles abióticos más importantes para regular la comunidad biológica en un río están las alteraciones que ocasiona la forma en que se recarga el sistema (régimen hídrico). El sistema del río Moctezuma presenta un régimen hídrico con una elevada tasa de recarga de los caudales, producto de las lluvias torrenciales de la región. De esta forma se presenta una elevación del caudal y del flujo en pocos días, lo que conlleva a un arrastre indiscriminado de la ic- 
tiofauna, especialmente de las especies de pequeño tamaño, quedando solo, en su lugar de origen, los individuos que estaban refugiados al momento de la crecida. Este fenómeno fue muy acusado en los ríos con alto manejo humano de sus caudales, en el sentido de que las represas mantenían la corriente del agua lenta y la permanencia de más especies de peces que en los tramos sin retenciones de agua. La comparación de métodos de medición de la diversidad $\beta$ reveló que en esta cuenca hay recambio de especies gradual y discontinuo, con tendencia a incrementarse el cambio gradual.

Para realizar cualquier propuesta de conservación, o planes de gestión de las ecorregiones de la cuenca Moctezuma, se debe entender que la relación de la diversidad ictiofaunística con el medio físico en general depende de la escala espacial a la que se trabaje, dado que los factores que la determinan están subrogados; por ejemplo, puede jerarquizarse la zonificación de la cuenca en dos escalas: 1) Regional: los factores más importantes para la distribución de la fauna acuática y la estructura de la comunidad son el tipo de ecosistema (río, arroyo, lago, manantial) y el tipo de ecotopo (lótico/léntico); 2) Local: la velocidad de corriente, el microhábitat, la profundidad y la vegetación determinan la ictiofauna de cada elemento. La altitud también se manifiesta como un factor limitante en la dispersión de la ictiofauna en ambas escalas (Morales-Ortiz \& Gutiérrez-Yurrita, 2000). Debido a que los procesos bióticos y abióticos generan patrones de estructura comunitaria en los peces, y ambos, procesos y patrones son dependientes de la escala, permiten reconocer regiones ecológicas a lo largo de la cuenca, delimitadas por la distribución de grupos de especies de peces (Fig. 5). De igual forma, permite conocer los subrogados ecológicos para plantear nuevas formas de conservación de las cuencas y sobretodo, de las metacuencas (cuencas conectadas en red).

En síntesis, la integración de la información conduce a observar 1) que los patrones de distribución de los peces de esta cuenca se ven influidos por factores como la cantidad y calidad de agua (régimen hídrico), así como por la fluctuación estacional del sistema hidrológico en general (hidroperiodo). 2) que la mayoría de las es- pecies pueden mantener sus poblaciones bajo una gran variedad de condiciones fisicoquímicas, siendo capaces de interactuar con una alta diversidad de especies y colonizar hábitats disponibles. Estas interacciones tienden a formar conjuntos faunísticos particulares; y 3) que la comunidad íctica de la cuenca Moctezuma, aunque es propia de un sistema montañoso, tolera grandes cambios de altitud, localizándose casi a nivel del mar. Bajo este panorama, los planes de acción que podrían aplicarse para remediar los impactos señalados, requerirían trabajar a partir del manejo ecosistémico de metacuencas y mediante una gestión coordinada de los recursos económicos públicos y privados. A través de una zonificación del medio como la planteada en la figura 2 y jerarquizada, puede lograrse un desarrollo sustentable, no sólo para la parte queretana de la cuenca del río Moctezuma, sino para la ecozona del sistema acuático del Centro de México MoctezumaPánuco; por lo que deben desarrollarse diversos programas sectoriales y horizontales, sin olvidar que hay una interacción entre las diferentes escalas en las cuales se actuará (Gutiérrez-Yurrita $e t$ al., 2004). A partir de los programas sectoriales, pueden diseñarse los caudales ecológicos de cada subcuenca, para emular su hidroperiodo y régimen hídrico natural (Gutiérrez-Yurrita, 2012).

\section{AGRADECIMIENTOS}

Los autores queremos expresar un especial agradecimiento al Dr. Salvador Contreras-Balderas por la paciencia y los comentarios que nos hizo sobre algunas determinaciones taxonómicas e intervalos de distribución de las especies, en especial las del género Notropis.

\section{BIBLIOGRAFÍA}

ABELL, R. A., D. M. OLSON, E. DINERSTEIN, P. T. HURLEY, J. T. DIGGS, W. EICHBAUM, S. WALTERS, W. WETTENGEL, T. ALLNUTT, C. LOUCKS, \& P. HEDAO. 2000. Freshwater ecoregions of North America. A conservation assessment. Island Press, Washington, Estados Unidos. 
ALCOCER, J. \& F. BERNAL. 2010. Limonolgy in Mexico. Hydrobiologia, 644: 15-68.

ALONSO-EGUÍA LIS, P., E. GONZÁLEZ-SORIANO \& P. J. GUTIÉRREZ-YURRITA. 2002. Listado y distribución de los odonatos de la Cuenca del Río Moctezuma, Centro-Occidente de México (Insecta: Odonata). Folia Entomologica Mexicana, 41(3): 347-357.

ÁLVAREZ, V. J. 1970. Peces mexicanos (claves). Secretaría de industria y Comercio. Dirección General. México.

— 1972. Ictiología Michoacana V. Origen y distribución de la ictiofauna dulceacuícola de Michoacán. Anales Escuela Nacional de Ciencias Biológicas, 19: 155-161 + 1 lámina.

ARREDONDO-FIGUEROA, J. L. \& M. GUZMÁNARROYO. 1985. Actual situación taxonómica de las especies de la tribu Tilapiini (Pisces: Cichlidae) introducidas en México. Anales del Instituto de Biología, 56. Serie Zoológica (2): 555-572.

BRUNO, D., D. SÁNCHEZ, J. A. CARBONELL, F. PICAZO, J. VELASCO \& A. MILLÁN. 2012. Predicting the Richness of Aquatic Beetles and Bugs in a Semi-Arid Mediterranean Region. Limnetica, 31 (1): 23-36.

BURR, M. B. \& L. R. MAYDEN. 1992. Phylogenetics and North American Freshwater Fishes. On Systematics, Historical Ecology, and North American Fishes. Stanford University Press, Estados Unidos.

CASTRO-AGUIRRE, J. H. ESPINOSA y J. SCHMITTER-SOTO. 1999. Ictiofauna estuarina lagunar y vicaria de México. Noriega-Limusa-IPN, México.

CHASE, J. M \& M. A LEIBOLD. 2003. Ecological Niches: Linking Classical and Contemporary Approaches. University of Chicago Press.

DE BUEN, F. 1947a. Investigaciones sobre ictiología mexicana I. Catálogo de los peces de la región Neártica en el suelo mexicano. Anales del Instituto de Biología, UNAM. XVIII: 256-292.

- 1947b. Investigaciones sobre ictiología mexicana II. Repartición geográfica sobre suelos mexicanos de las familias típicamente dulceacuícolas. Anales del Instituto de Biología, UNAM. XVIII: 293-343.

DE BUEN, F. 1947c. Investigaciónes sobre ictiología mexicana III. La ictiofauna de los alrededores de la Ciudad de Querétaro. Anales del Instituto de Biología, UNAM. XVIII: 533-537.

DE LA VEGA-SALAZAR, M. 2006. Estado de conservación de los peces de la familia Goodeidae
(Cyprinodontiformes) en la mesa central de México. Revista de Biología Tropical, 54(1): 163-177.

DEL POZO, R., M. FERNÁNDEZ \& C. FERNÁNDEZ. 2012. Composición de las comunidades de macrófitos y establecimiento del estado de conservación de charcas y lagunas de la Depresión del Duero (Noroeste de España) en base a criterios botánicos. Limnetica, 31 (1): 47-58.

ESPINOSA, H. D. GASPAR \& M. FUENTES. 1993. Listado faunístico de México. III. Los peces dulceacuícolas mexicanos. Instituto de BiologíaUNAM.

GUTIÉRREZ-YURRITA, P. J. 2004. The Use of the Crayfish Fauna in México: Past, Present... and Future? Freshwater Crayfish, 14: 45-51.

-2011. How to Restore Riverbanks in Natural Ecosystems with Many People Using Ecological Services at the Same Time? Chemical Engineering and Applications, 23: 15-20.

-2012. A Reflection on the Importance of Estimating Environmental Flows in Seasonal Rivers. The Case of Rivers in Central Mexico. Environment, Energy \& Biotechnology, 33: 55-59.

GUTIÉRREZ-YURRITA, P. J. \& C. MONTES. 1999. Bioenergetics and Phenology of Reproduction of the Introduced Red Swamp Crayfish Procambarus clarkii in Doñana National Park (Spain), and Implications for Management. Freshwater Biology, 42: 561-574.

GUTIÉRREZ-YURRITA, P. J. \& J. A. MORALESORTIZ. 2002. Ecophysiological Races of Cambarellus spp. in Querétaro, Central México. The Beginning of a New Species? Freshwater Crayfish, 13: $175-185$.

GUTIÉRREZ-YURRITA, P. J. \& M.Á., LÓPEZFLORES. 2011. Reflexiones ambientalistas sobre los criterios para proponer espacios naturales protegidos: hacia una nueva categoría de conservación, el Paisaje metafísico (o espiritual). Revista Aranzadi de Derecho Ambiental, 20(2): 19-42.

GUTIÉRREZ-YURRITA, P. J. \& L. M. GARCÍA \& C. MONTES. 2013. Ecological Design of a Crayfishing Program to Control Population Density of the Invasive Species Procambarus clarkii in Doñana National Park Freshwater Marsh (Spain). Hydrobiologia (en prensa).

GUTIÉRREZ-YURRITA, P. J., J. A. MORALESORTIZ, A. OVIEDO \& C. RAMÍREZ-PÉREZ. 2002. Distribution, Spread, Habitat Characterisation and Conservation of the Crayfish Species 
(Cambaridae) in Querétaro (Central México). Freshwater Crayfish, 13: 288-297.

GUTIÉRREZ-YURRITA, P. J., P. ALONSO, S. HURTADO, A. MORALES, E. BAUTISTA, C. PEDRAZA \& A. LÓPEZ-ROMERO. 2004. Estudios ecosistémicos en la Reserva de la Biosfera Sierra Gorda, Querétaro. Memorias del Primer encuentro de Investigación sobre la Sierra Gorda. UNDP-CONANP. 279-298. México.

HAMMER, Ø., HARPER, D.A.T., \& P. D. RYAN, 2001. PAST: Paleontological Statistics Software Package for Education and Data Analysis. Palaeontologia Electronica 4(1): 9 pp. http://palaeoelectronica.org/2001_1/past/issue1_01.htm

HOLOMUZKI, J. R. 2000. Taxon-Specific Responses to High-Flow Disturbance in Streams: Implications for Population Persistence. Journal of the North American Benthological Society, 19: 670679.

HURTADO, S., F. GARCÍA-TREJO \& P. J. GUTIÉRREZ-YURRITA. 2005. Importancia ecológica de los macroinvertebrados bentónicos de la subcuenca del río San Juan, Querétaro, México. Folia Entomológica Mexicana, 44(3): 271-286.

MAGURRAN, A. 1989. Diversidad ecológica y su medición. Vedrá, España.

MCMARIGAL, K. S. CUSHMAN y S. STAFFORD. 2000. Multivariate Statistics for Wildlife and Ecology Research. Springer. Estados Unidos.

MILLER, R. R. 1982. Pisces. En: S. H. HURBERT \& A. VILLALOBOS-FIGUEROA (Eds). Aquatic Biota of México, Central America and the West Indies: Being a Compilation of Taxonomic Bibliographies for the Fauna and Flora of Inland Water of Mesoamerica and the Adjacent Caribbean Region. San Diego, San Diego State University.

- 1986. Composition and Derivation of the Freshwater Fish Fauna of México. Anales de la Escuela Nacional de Ciencias Biológicas IPN, 30: 121153.

MILLER, R. R. \& M. SMITH. 1986. Origin and Geography of the Fishes of Central México. En: The zoogeography of North American Freshwater Fishes. Hocutt, C. H. y O. Wiley (eds.): 487-517. John and Wiley and Son Inc. New York, Estados Unidos.

MORALES-ORTIZ, J. A. \& P. J. GUTIÉRREZ-YURRITA. 2000. Observaciones sobre la distribución de Astyanax mexicanus (Filippi) 1854 (Characidae) en las cuencas de los ríos Moctezuma y Tampaón, Centro de México. Memorias del VII Congreso Nacional de Ictiología: 72-73.

NOM-059-SEMARNAT, 2010. Norma Oficial Mexicana publicada en el Diario Oficial de la Federación.

ODLING-SMEE, F. J., K.N LALAND \& M. W. FELDMAN. 2003. Niche Construction: the Neglected Process in Evolution. Princeton University Press. Princeton, NJ.

POWER, M., J. STOUT, C. CUSHING, P. HARPER, R. HAUER, W. MATTHEWS, P. MOYLE, B. STATZNER \& W. BARDGEN. 1988. Biotic and Abiotic Controls in River and Stream Communities. Journal of the North America Benthological Society, 7(4): 456-479.

RAVENTÓS, J. J. SEGARRA \& M. F. ACEVEDO. 2005. Modelos de metapoblaciones y de la dinámica espacio temporal de comunidades. Publicaciones de la Universidad de Alicante, España.

SCHULTZ, R. J. \& R. R. MILLER. 1971. Species of the Poecilia sphenops complex (Pisces: Poeciliidae) in México. Copeia, 2: 282-290.

TAYLOR, J. N \& R. R. MILLER. 1983. Cichlid Fishes (genus Cichlasoma) of the río Pánuco, Eastern México, with Description of a New Species. Occasional Papers of the Museum of Natural History. University of Kansas. Estados Unidos.

TILMAN, D. 1994. Competition and Biodiversity in Spatially Structured Habitats. Ecology, 75:2-16.

VANNOTE, R. L., W. G. MINSHALL, K. W. CUMMINS, J. R. SENADEL \& C. CUSHING. 1980. The River Continuum Concept. Canadian Journal of Fisheries and Aquatic Sciences, 37: 130-137.

WHITTAKER, R. H. 1975. Communities and Ecosystems. 2nd Edition. MacMillan Pub. Co. Nueva York, Estados Unidos. 